\title{
Pancreatic function testing - valuable but underused
}

\author{
Chris Forsmark MD¹, Paul C Adams MD², Editor-in-Chief
}

$D^{\prime}$ Chris Forsmark is Chief of the Division of Gastroenterology, Hepatology and Nutrition at the University of Florida (Gainesville, Florida, USA). His clinical and clinical research interests are in pancreatic diseases, particularly chronic pancreatitis and the measurement of pancreatic function.

PA: Can you briefly review the history of pancreatic secretion studies to assess pancreatic function?

CF: A wide variety of tests have been developed to measure pancreatic function. These often began as clinical research tools to study pancreatic physiology, but subsequently have evolved into diagnostic tests. Pancreatic function can be measured in many different ways. We often divide these tests into those that directly measure pancreatic secretion and those that measure function indirectly by assessing the secondary effects resulting from a lack of digestive enzymes. These tests have also been grouped into invasive tests requiring a tube or an endoscope, and noninvasive tests. The principal direct test of pancreatic function is a hormonal stimulation test. In this test, an oroduodenal tube is placed and used to collect pancreatic secretions. A hormonal secretagogue (either secretin or cholecystokinin [CCK]) is given, and pancreatic secretions are collected via the tube and analyzed. When CCK is administered, pancreatic output of lipase is measured; when secretin is administered, pancreatic output of bicarbonate is measured. This type of testing is not standardized and each of the few centres that perform the test has had to define a protocol and normal ranges.

Another approach to pancreatic function testing is to measure the effect (or lack thereof) of the action of pancreatic enzymes - an indirect pancreatic function test. Measuring fecal fat is one such measurement. There have also been literally hundreds of variations of these indirect tests with the same approach:

1. Administer a substrate that requires pancreatic enzymes for digestion;

2. Measure the metabolites of the substrate in stool, urine, blood or breath; and

3. Establish ranges for patients with chronic pancreatitis and those without.

All of these tests share one major drawback - they will usually not be positive until the pancreas has developed exocrine insufficiency - a process that takes years. They are, therefore, likely to be quite inaccurate in the early or less-advanced stages of disease. PA: How useful are fecal elastase studies?

CF: This test measures the concentration of elastase in stool. In patients who have advanced chronic pancreatitis, the delivery of elastase to the duodenum is reduced. Elastase can travel through the small bowel and colon without being metabolized or destroyed, and can be measured in the small bowel. Fecal elastase levels of less than $100 \mu \mathrm{g} / \mathrm{g}$ of stool are present in patients with advanced chronic pancreatitis. The test can be performed in patients already on enzyme replacement therapy because these are generally porcine products and the test measures human elastase. The test is generally positive in subjects with advanced disease (ie, exocrine insufficiency) but inaccurate in less advanced disease.

PA: There had been some interest in measuring metabolites of para-aminobenzoic acid (PABA) compounds degraded by pancreatic enzymes.

CF: Presently, this test is generally only of historical interest. Bentiromide was administered by mouth and could be metabolized by pancreatic chymotrypsin which, in turn, liberated PABA. The PABA could be absorbed and eventually excreted in the urine. Low levels of PABA detected in the urine implied low levels of pancreatic chymotrypsin and, hence, chronic pancreatitis. The test is inaccurate in less-advanced disease and the substrate is no longer available for clinical use.

PA: How useful are imaging studies for estimating pancreatic function?

CF: There are many imaging tests that can provide useful information on the pancreas and the presence of chronic pancreatitis. These include computed tomography (CT), magnetic resonance imaging (MRI)/MR cholangiopancreatography (MRCP), endoscopic ultrasound (EUS) and others. The only imaging test that is currently being used in attempts to measure pancreatic function is MRI with MRCP and secretin injection. In this test, an MRI and MRCP are obtained, then the hormonal secretagogue is administered. On sequential MRCP images, fluid secreted by the pancreatic duct and bile duct can be imaged on MRI, and the relative amount of fluid produced can be estimated. Administering secretin makes the pancreatic duct more visible on MRCP, which enhances its diagnostic sensitivity. To this point, however, there are no data to support

${ }^{1}$ Division of Gastroenterology, University of Florida, Gainesville, Florida, USA; ${ }^{2}$ University Hospital, London, Ontario

Correspondence: Dr Chris Forsmark, Division of Gastroenterology, Hepatology and Nutrition, University of Florida, Gainesville, Florida, USA.

Telephone 352-392-2877, fax 352-392-3618, e-mail chris.forsmark@medicine.ufl.edu

Accepted for publication October 22, 2008 
the hypothesis that estimating or quantifying the fluid output at MRCP is accurate as a diagnostic test, although several centres are studying the technique.

PA: Can you describe an endoscopic pancreatic function test? CF: Patients are sedated in the standard fashion. Synthetic human secretin is administered intravenously as a bolus at a dose of $0.2 \mu \mathrm{g} / \mathrm{kg}$ body weight. The endoscopy is performed in the standard fashion, with attention given to suctioning all existing fluid from the stomach and duodenum. The endoscope is positioned in the duodenum, opposite the major papilla. Aliquots of duodenal fluid are collected at $15 \mathrm{~min}, 30 \mathrm{~min}, 45 \mathrm{~min}$ and 60 min after injection of secretin. These samples are sent to the hospital laboratory to measure bicarbonate concentration. The highest value of the four samples is considered the peak bicarbonate concentration. A peak bicarbonate level of less than $80 \mathrm{mEq} / \mathrm{L}$ is considered abnormal. This test has very high sensitivity but low specificity. It is most useful for ruling out disease. In other words, a negative test essentially rules out chronic pancreatitis, while a positive test requires confirmation (usually with EUS).

These techniques have been developed and standardized by Darwin Conwell, of the Cleveland Clinic and Brigham and Women's Hospital (USA). The advantage of hormonal stimulation tests is that they measure a decrease in maximum stimulated secretory capacity, which begins to decline before the pancreas is extensively damaged. In other words, the results of these tests become abnormal before other tests that detect features of more advanced damage to the pancreas become abnormal (such as CT or endoscopic retrograde cholangiopancreatography).

PA: How do you dose pancreatic enzymes?

CF: Pancreatic enzymes can be used to treat pancreatic pain or to treat exocrine insufficiency with maldigestion. The use of enzymes to treat pain is predicated on the fact that some patients with chronic pancreatitis appear to have very high levels of CCK in the blood, which produces excessive stimulatory drive on the pancreas. Delivering serine proteases (from enzymes) can interfere with CCK release by destroying a releasing factor produced in the duodenum and decrease this hyperstimulation. This requires using an enzyme formulation that can deliver these proteases to the feedback-sensitive portion of the small intestine (the duodenum and most proximal jejunum). Only nonenteric-coated preparations are able to accomplish this. Enteric-coated preparations release their products further downstream. The nonenteric-coated preparations can be destroyed by gastric acid; consequently, patients need to be cotreated with either an $\mathrm{H}_{2}$ receptor antagonist or proton pump inhibitor. The typical dose for treating pain is the equivalent of four pancrelipase tablets (Viokase-16, Axcan Pharma Inc, USA) with meals and at night, along with acid suppression. This therapy is most effective in patients with less-advanced chronic pancreatitis (ie, without exocrine or endocrine insufficiency).

The management of exocrine insufficiency usually uses the enteric-coated preparations but the nonenteric-coated agents work equally well. It has been estimated that approximately 30,000 IU of lipase be delivered with each meal to reduce steatorrhea to insignificant levels. Remember, most preparations in the United States and Canada are dosed in United States Pharmacopeia (USP) units, which are less than IU (1 IU $=3$ USP). In adults with chronic pancreatitis, we do not typically try to completely eliminate steatorrhea; therefore, doses of 30,000 USP to 60,000 USP are usually adequate, although some patients may need more. This translates into the equivalent of three pancrelipase capsules (Creon 20, Solvay Pharmaceuticals, USA) with meals and one to two with snacks, although some patients require higher doses. These enteric coated preparations do not require acid suppression. If diarrhea disappears and patients maintain their weight, I do not routinely check fecal fat levels to assess efficacy. I pay special attention to osteoporosis, which is common in these patients, and put all of them on calcium and vitamin D supplementation. If enzyme therapy is not effective, it is prudent to search for alternative explanations. Among alternative explanations, small bowel bacterial overgrowth is the most common. PA: Why is it important to measure pancreatic exocrine function?

CF: Many patients with chronic pancreatitis are easily diagnosed with widely available tests such as CT, EUS or MRI. These patients usually have fairly longstanding and advanced disease. Another group of patients presents to medical attention for evaluation of a chronic abdominal pain syndrome. In many of these patients, tests such as CT or MRI are normal. Despite this, the patient is diagnosed as having chronic pancreatitis (often called 'small-duct' or 'minimal-change' chronic pancreatitis) based on the characteristics of the pain, or the fact that serum amylase or lipase may have been elevated at some point during the illness. These patients may have chronic pancreatitis but often have other conditions including motility disorders and visceral hyperalgesia. I often see many such patients in my outpatient clinic and, unfortunately, they have often been treated with high doses of narcotics and have undergone risky and invasive testing (such as endoscopic retrograde cholangiopancreatography or celiac plexus block). The measurement of pancreatic function via a traditional or endoscopic direct pancreatic function test is the best way to determine whether they actually have 'minimal-change' chronic pancreatitis. In many of these patients, the test is normal, which allows me to remove the label of 'chronic pancreatitis' and to help them avoid invasive tests and risky therapies in the future. 


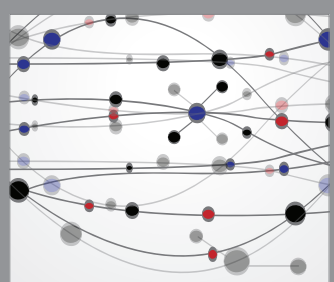

The Scientific World Journal
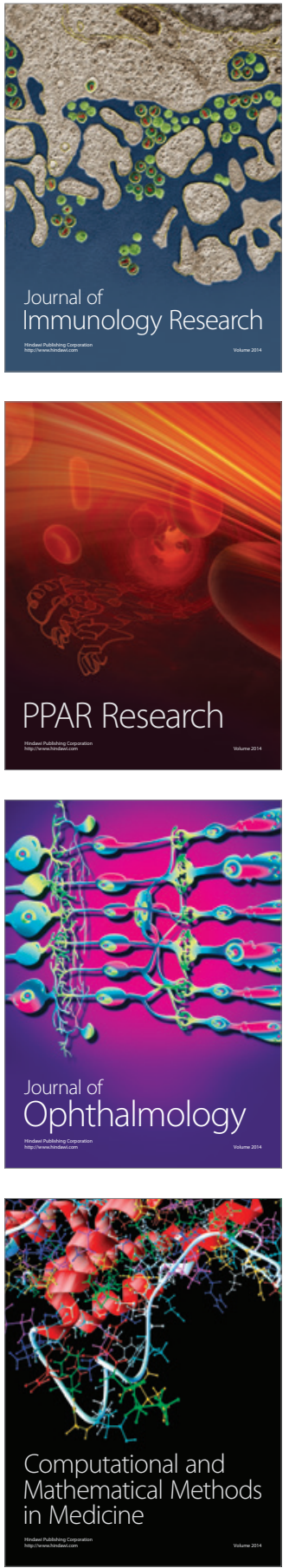

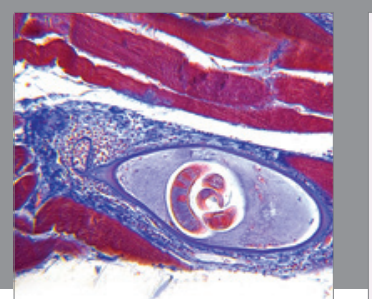

Gastroenterology Research and Practice

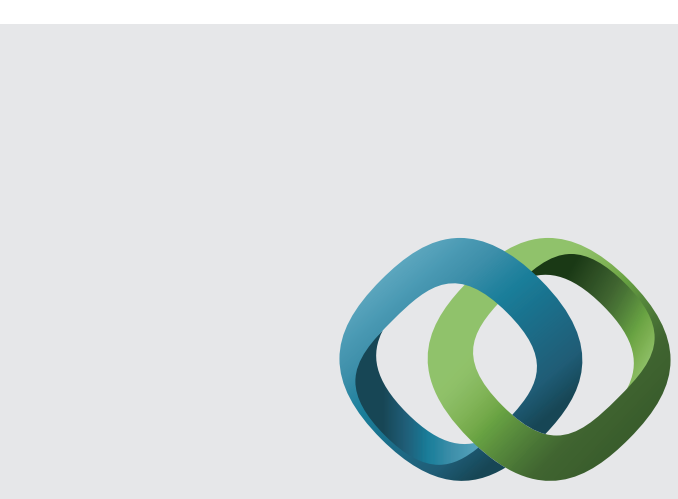

\section{Hindawi}

Submit your manuscripts at

http://www.hindawi.com
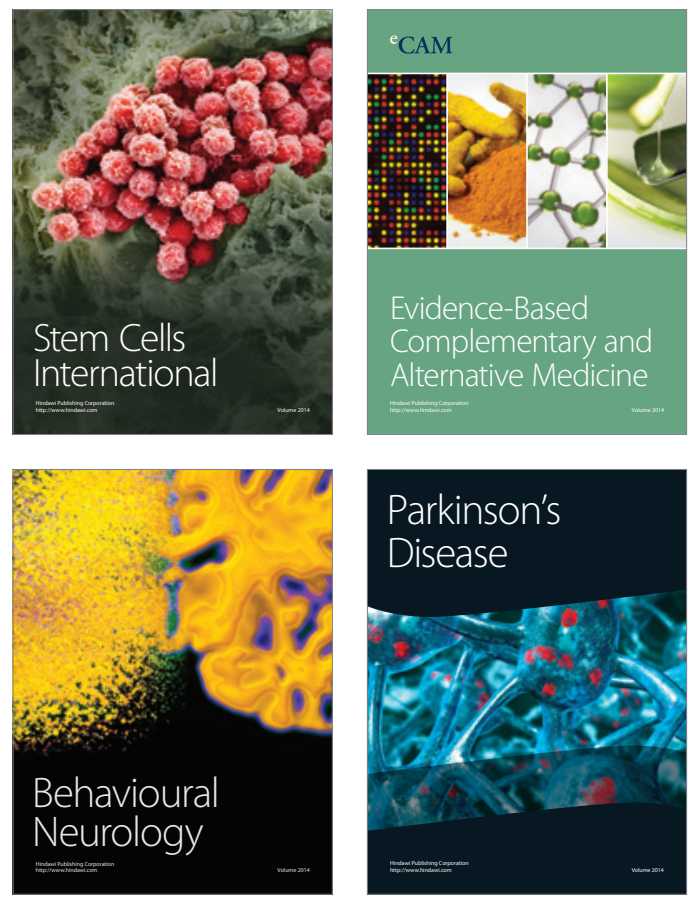
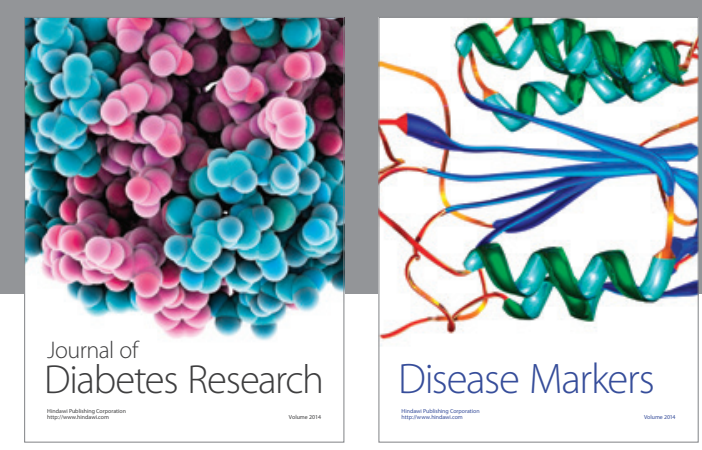

Disease Markers
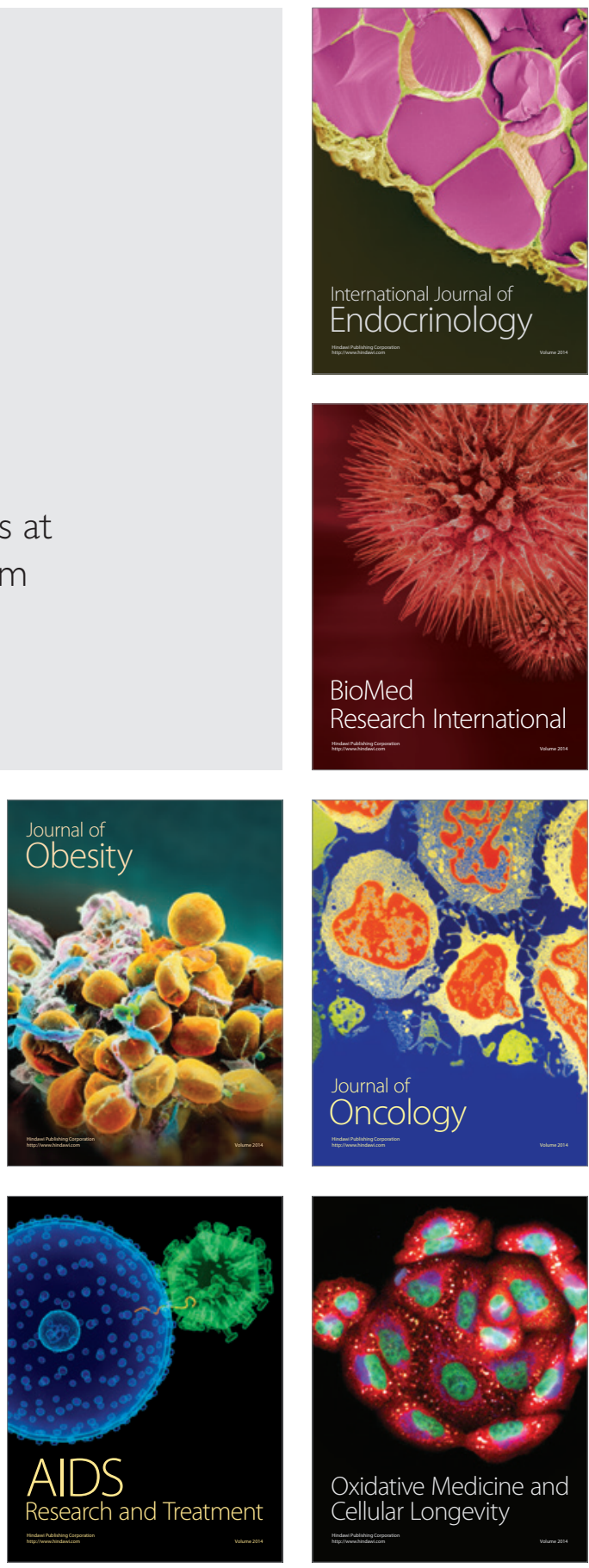